\title{
Local Heat Transfer Measurement in Pool Boiling Using High-speed IR Thermography
}

\author{
by T. Yabuki*, T. Tanaka*, K. Miyzaki
}

*Kyushu Institute of Technology, Department of Mechanical and Control Engineering, 1-1 Sensui-cho, Tobata-ku, Kitakyushu, Fukuoka 804-8550, Japan, yabuki.tomohide556@mail.kyutech.jp

\begin{abstract}
A high-resolution temperature measurement technique is required for the detailed characterization of fundamental heat transfer processes in pool boiling heat transfer. Fundamental heat transfer processes in pool boiling were observed through the wall temperature distribution measurement using high-speed IR thermography in this study. The measured temperature clearly shows the presence of evaporation and dry-out of microlayer, and rewetting of the dried area. The local heat transfer in the boiling process was evaluated through the 3-D transient heat conduction calculation using the measured temperature as the surface boundary condition.
\end{abstract}

\section{Introduction}

Nucleate boiling is the most efficient heat transfer mode, and has been utilized in a variety of heat transfer systems. The boiling includes the fundamental heat transfer phenomena such as microlayer evaporation beneath the bubble, threephase contact-line heat transfer, evaporation of superheated liquid surrounding the bubble, forced convection induced by the bubble motion, transient heat conduction in liquid after a bubble departure. Better observation and understanding of the fundamental heat transfer phenomena are essential to construct a mechanistic boiling heat transfer model. It has been, however, difficult for commonly-used sensors to precisely measure fundamental heat transfer phenomena at a small spatiotemporal scale, and therefore physical mechanisms of the fundamental phenomena have remained unclear. Recently, high-resolution temperature measurement techniques have been developed and enable the direct measurement of the fundamental heat transfer phenomena of boiling. The boiling mechanisms have been investigated with high resolution measurement techniques including high-speed IR thermometry [1] and MEMS thermometry [2]. In this study, high-speed IR thermography is used for detailed observation of local heat transfer characteristics in pool boiling of water.

\section{High-resolution measurement of temperature distribution beneath boiling bubble}

The temperature distribution of the heated surface was measured using the high-speed camera (Telops, FAST $\mathrm{M} 3 \mathrm{k}$ ) at a framing rate of $5 \mathrm{kHz}$ from the bottom of the heated wall. The sapphire substrate with a thickness of $0.6 \mathrm{~mm}$ and a size of $20 \times 20 \mathrm{~mm}^{2}$ was used as a heated wall. The titanium thin film was sputtered as a heater and also a radiator. The titanium thin film heater covers the area of $10 \times 10 \mathrm{~mm}^{2}$ at the centre of the sapphire substrate. In the boiling experiment, the sapphire substrate is placed at the bottom of the boiling chamber and the water as a boiling liquid is heated to saturation temperature at an atmospheric pressure using immersed sheathed heater.

Figure 1 shows an example of the measured temperature distribution (bottom) and the schematic drawings of the bubble behaviour (upper) read from the temperature distribution. The time zero corresponds to the nucleation time of the bubble. The thin liquid film called microlayer is formed under the bubble. The microlayer is thin as on the order of $1 \mu \mathrm{m}$, and intensive evaporation of the microlayer happens under the bubble. The microlayer evaporation causes quick temperature decrease. The microlayer dries out and the dry-out causes sudden deterioration of the surface heat transfer coefficient. This dry-out results in temperature increase. Combination of the microlayer evaporation and the dry-out outside that causes ring shape temperature decrease.

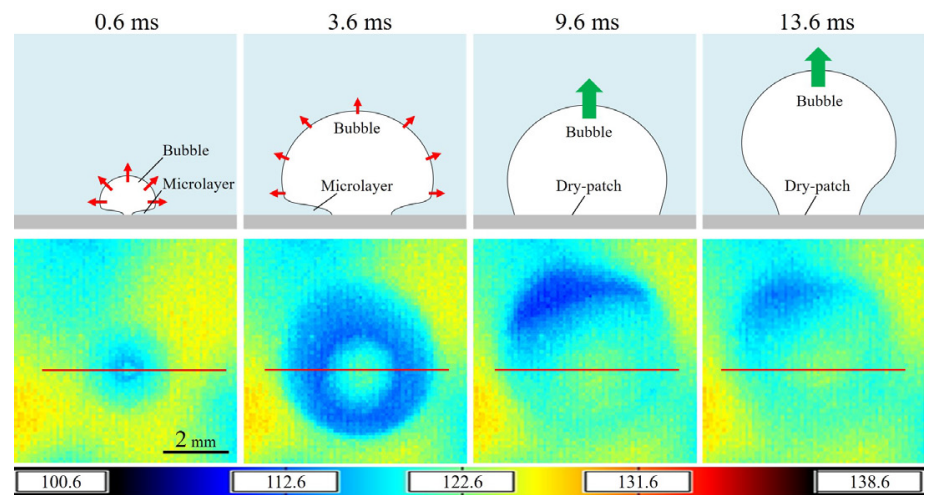

Figure 1. Temperature distribution under a bubble (bottom) and schematic drawings of bubble behaviour (upper). 


\section{Local heat transfer beneath boiling bubble}

The local heat flux distribution was calculated through the three dimensional transient heat conduction calculation in the sapphire substrate. The measured temperature in Fig. 1 was used as the boundary condition at the heated surface. The temperature distribution inside the substrate can be calculated through the heat conduction simulation and the heat flux distribution on the heated surface was calculated from the temperature gradient at the surface. Figure 2 shows the heat flux distribution on the red line in Fig. 1 in the bubble growth process (left) and in the bubble detachment process (right). The x-axis in Fi. 2 corresponds to the distance from the bubble nucleation site. The microlayer evaporation causes positive heat flux peak over $1 \mathrm{MW} / \mathrm{m}^{2}$ in the bubble growth process. The heat flux peak expands outward with time due to the expansion of the microlayer region. On the other hand, the dry-out of the microlayer causes the decrease in heat flux. The low heat flux region expands outward due to the expansion of the dry-patch. In the bubble detachment process, it can be predicted that the contact line advances to the bubble centre and the rewetting of the dry-patch enhances heat transfer. However, the significant positive heat flux peak cannot be observed in the bubble detachment process. Figure 3 shows heat flow under the bubble calculated by spatial integration of the heat flux distribution. The bubble detached from the surface at $18.4 \mathrm{~ms}$. The heat flow most increases during the microlayer evaporation and the heat transfer enhancement is not observed in the bubble detachment process. This indicates that the microlayer evaporation plays an important role in the wall heat transfer during the boiling of water.

(a)

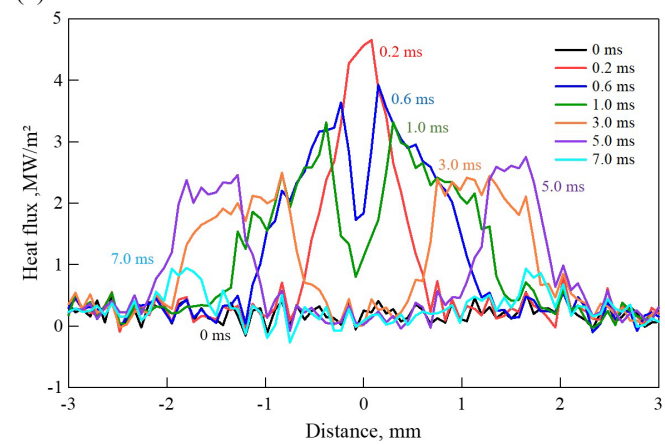

(b)

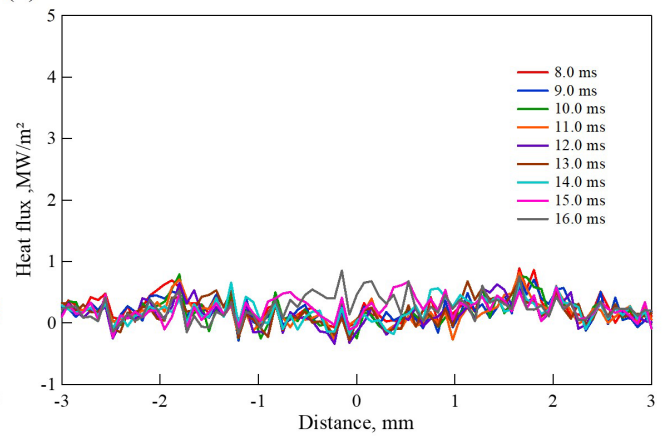

Fig. 2 Local heat flux distribution in the bubble growth (left) and detachment (right) processes.

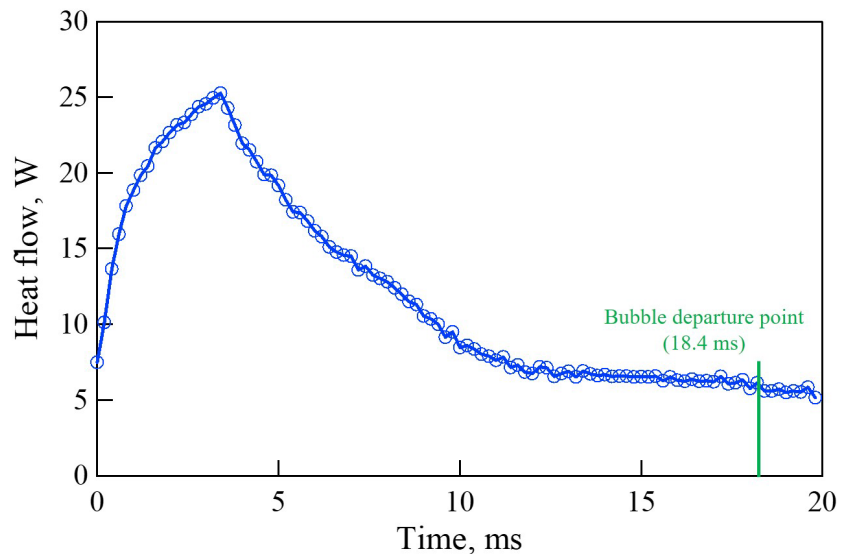

Fig. 3 Heat flow transferred in isolated bubble event.

\section{REFERENCES}

[1] C. Gerardi, J. Buongiorno, L. Hu, T. McKrell, Study of bubble growth in water pool boiling through synchronized, infrared thermometry and high-speed video, Int. J. Heat Mass Transfer 53 (2010) 4185-4192.

[2] T. Yabuki, O. Nakabeppu, Heat transfer mechanisms in isolated bubble boiling of water observed with MEMS sensor, Int. J. Heat Mass Transfer 76 (2014) 286-297. 